\title{
The Biosynthetic Gene Cluster for the $\beta$-Lactam Antibiotic Tabtoxin in Pseudomonas syringae
}

\author{
Thomas G. Kinscherf, David K. Willis
}

Received: August 24, 2005 / Accepted: November 11, 2005

(C) Japan Antibiotics Research Association

\begin{abstract}
DNA sequence analysis revealed that the biosynthetic genes of the unusual $\beta$-lactam antibiotic tabtoxin reside at the att site adjacent to the lys $C$ tRNA gene in Pseudomonas syringae BR2. ORFs encoded within the region included ones with similarity to $\beta$-lactam synthase and clavaminic acid synthase, as well as amino acid synthesis enzymes. Novel ORFs were present in a portion of the biosynthetic region associated with a toxin hypersensitivity phenotype. Tabtoxin resistance was associated with a fragment containing a major facilitator superfamily (MFS) transporter gene.
\end{abstract}

Keywords $\beta$-lactam, antibiotic, integrase, synthase, clavam, MFS multidrug-resistance

Decades of research and development have led to the invention of multiple generations of $\beta$-lactam antibiotics that remain to the current day in the forefront of the fight against human infectious disease. Some therapeutic $\beta$ lactam antibiotics are derived by chemical modification of naturally occurring mature and precursor forms, thereby making an understanding of the underlying biosynthetic pathways available in nature of great interest. Tabtoxinine$\beta$-lactam (TBL) is a novel peptide $\beta$-lactam antibiotic that is produced by the cleavage of its dipeptide precursor tabtoxin [1, 2] (Fig. 1). The antibiotic is usually associated with bacteria of the Pseudomonas syringae species group causal to several types of chlorotic plant disease. While most $\beta$-lactam antibiotics exert their antimicrobial

D. K. Willis (Corresponding author), T. G. Kinscherf: USDA/ARS Vegetable Crops Research Unit and Department of Plant Pathology, University of Wisconsin - Madison, Madison WI 53706, E-mail: dkwillis@wisc.edu

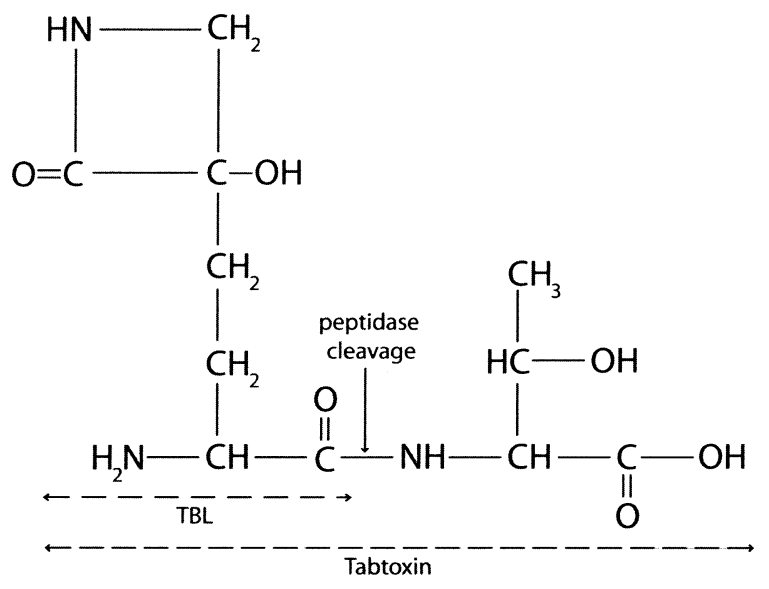

Fig. 1 The dipeptide precursor tabtoxin is processed by a peptidase to create the active antibiotic tabtoxinine- $\beta$ lactam (TBL).

influence through the inhibition of bacterial cell wall synthesis, TBL appears to irreversibly inhibit the enzyme glutamine synthetase, causing cells to become intoxicated by high levels of their own unprocessed ammonia [3, 4]. The genes encoding the pathway for both tabtoxin production and host resistance were originally cloned as a single cosmid insert from BR2, a Pseudomonas syringae strain causal to wildfire disease on bean [5]. Here we report the analysis of the DNA sequence of this cloned region.

Subcloned fragments of the cosmid pRTBL823 were sequenced using a combination of primer walking with synthetic primers derived from endogenous sequence within the insert DNA and transposon priming (GPS-1, New England Biolabs). Sequence of plasmid miniprep DNA (Qiagen) was generated using dye terminator kits (ABI) analyzed by capillary electrophoresis (GeneAnalyser $310, \mathrm{ABI})$. The sequence of overlaps between subclones was confirmed by sequencing with the original cosmid 
Table 1

\begin{tabular}{|c|c|c|c|}
\hline $\begin{array}{l}\text { ORF designation } \\
\quad \text { in Fig. } 1\end{array}$ & Gene name & Protein homology or similarity & $\begin{array}{c}\text { GenBank accession } \\
\text { Nnumber }\end{array}$ \\
\hline 1 & & phage integrase (XerC) & AAM77365 \\
\hline 2 & & virulence, unknown function & AAL99262 \\
\hline 3 & & prophage (fragment) & AAL92457 \\
\hline 4 & $\operatorname{tabP}$ & metallopeptidase & AAL99263 \\
\hline 5 & $\operatorname{tab} D$ & aat-like aminotransferase & AAM13981 \\
\hline 6 & $\operatorname{tab} B$ & $\begin{array}{l}\text { DapD, tetrahydrodipicolinate } \\
\text { N-succinyltransferase }\end{array}$ & P31852 \\
\hline 7 & $\operatorname{tab} A$ & LysA, diaminopimelate decarboxylase & AAB41802 \\
\hline 8 & tb/A & no matches in database & AAB25380 \\
\hline 9 & $\operatorname{tabC}$ & no matches in database & AAM75344 \\
\hline 10 & $t b / S$ & $\begin{array}{l}\beta \text {-lactam synthetase } \\
\quad(\text { AsnB, asparagine synthase) }\end{array}$ & AAM77668 \\
\hline 11 & $t b / C$ & $\begin{array}{l}\text { clavaminic acid synthetase } \\
\text { (TauD, taurine dioxygenase) }\end{array}$ & AA063153 \\
\hline 12 & $t b / D$ & $\begin{array}{l}\text { apparent fusion: GMC oxidoreductase- } \\
\text { GNAT acetyltransferase }\end{array}$ & AY744151 \\
\hline 13 & $t b / E$ & membrane protein ${ }^{a}$ & AAP13070 \\
\hline 14 & $t b / F$ & $\begin{array}{l}\text { D-ala D-ala ligase } \\
\qquad \text { (AccC, biotin carboxylase) }\end{array}$ & AAP13071 \\
\hline 15 & $t b / R$ & sugar transporter (MFS transporter) & AAP13072 \\
\hline 16 & & membrane protein ${ }^{a}$ & AA063154 \\
\hline 17 & & membrane protein ${ }^{a}$ & AAP14349 \\
\hline 18 & & unknown function & AAP13069 \\
\hline 19 & & unknown function & AAP14350 \\
\hline 20 & & unknown function & AAP14351 \\
\hline
\end{tabular}

${ }^{a}$ Membrane proteins defined as predicted using SOSUI algorithms

(http://sosui.proteome.bio.tuat.ac.jp/sosuiframe0.html).

pRTBL828 itself. Contig overlay and compilation was performed using the Wisconsin Package of the Genetic Computer Group (GCG). Comparative analysis of open reading frames (ORFs) within the sequence was performed on-line at the National Center of BioInformatics (NCBI) using the most current BLAST algorithms to scan the GENBANK non-redundant database. Tests for biological function were performed by cloning relevant fragment(s) in the mobilizable vehicle pRK415 and then transferring the constructs to an appropriate $P$. syringae host by triparental mating [6].

A total of 28,185 base pairs were sequenced from the tabtoxin biosynthetic region and the surrounding chromosome. The sequenced region was found to have a $\mathrm{G}+\mathrm{C}$ content of about $56 \%$ and was adjacent to the lys C tRNA gene, a locale that has been previously described as an att site for a variety of gene islands in genus Pseudomonas $[7 \sim 10]$. Sequence sampling of the chromosome on the distal side of the tRNA gene relative to the tabtoxin grouping indicated that gene organization there is essentially identical to P. putida, with the immediate gene neighbor being exs $B$. The sequenced portion of the gene island itself was logically broken down into four distinct blocks by presumed function and predicted direction of transcription:

\section{I) Gene Transfer Block}

The portion of the tabtoxin gene island closest to the lys $C$ tRNA gene contains genes associated with genetic dynamism in other species and genera of bacteria. The gene adjoining the tRNA gene encodes a full-length product (ORF 1, Fig. 2) with high similarity to XerC, a prototypical phage integrase (tyrosine recombinase). Such genes have often been found near the beginning of chromosome regions thought to result from the horizontal transfer of DNA into the current host $[7,8,10]$. The gene next to the 
Tabtoxin Biosynthetic Region

(28185 bp)

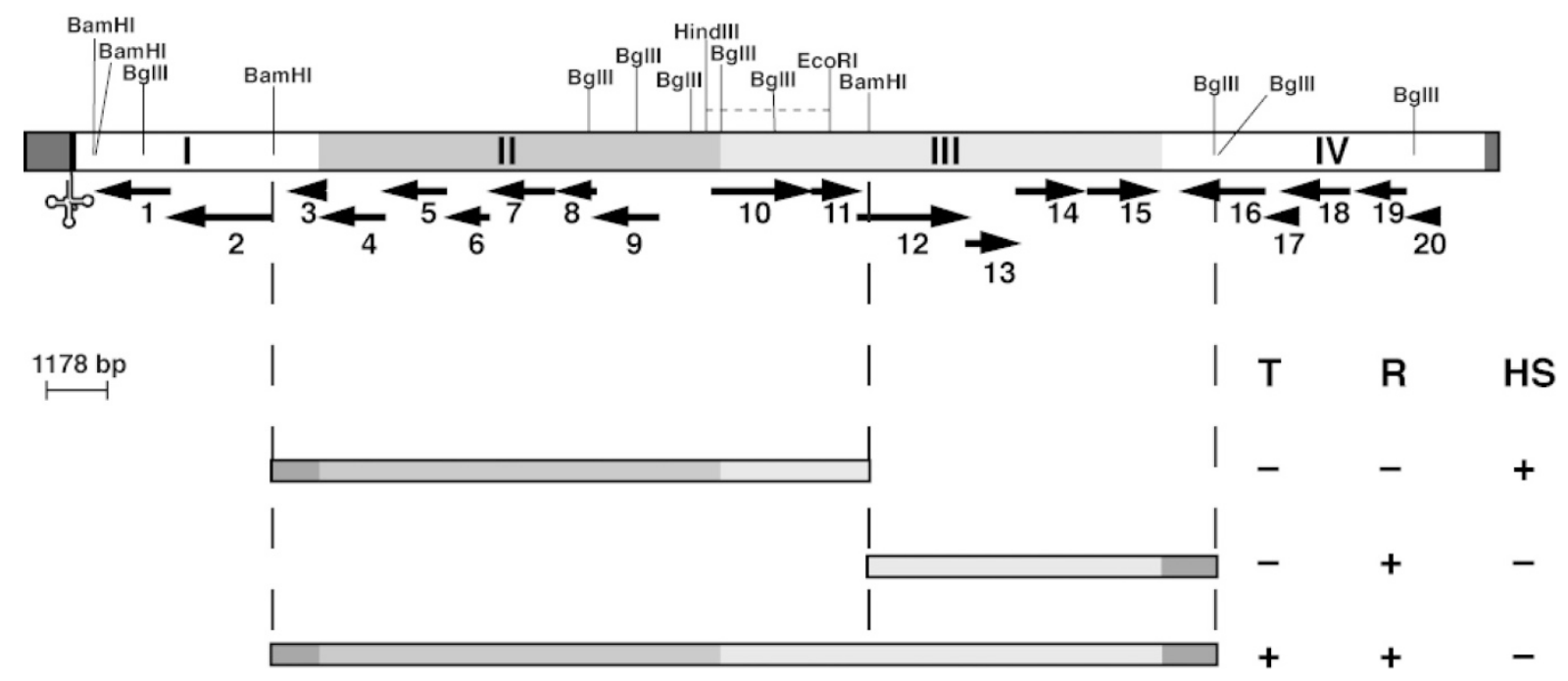

Fig. 2 The tabtoxin biosynthetic region is shown graphically, with the gene blocks numbered as described in the text. The location of the lysC tRNA is denoted by the cruciform; ORFs are shown as arrows pointing in the predicted direction of transcription relative to one another. Fragments and the respective toxin phenotypes associated with those fragments are shown below the whole region: toxin production as measured by bioassay with Escherichia coli (T); tabtoxinine- $\beta$-lactam resistance in bioassay (R); and hypersensitivity (HS, see Fig. 2) [5]. The EcoRI-Hindlll fragment containing the tb/S gene is shown as a dashed line.

integrase encodes a product of unknown function (ORF 2, Fig. 2) that has also been previously found in the genomes of a variety bacterial species. Studies with the dually plant and animal pathogenic $P$. aeruginosa strain PA14 found a highly similar ORF, there referred to as RL003, paired with a phage integrase fragment in a region designated the PAPI1 pathogenicity island [11]. In those studies, mutations in RL003 led to a decrease in both bacterial growth in plants and virulence in mice [11]. The final gene product (ORF 3, Fig. 2) in this block is similar to that of the yedK gene of Escherichia coli; this gene may also be phage related, with similarity to the gyfsy-2 prophage of Salmonella implicated in the horizontal transfer of virulence factors [12].

\section{II) Left Biosynthetic Block}

The darker shaded region of Fig. 2 contains the 'leftward' block of the tabtoxin biosynthetic genes, which is separated from the 'rightward' block of biosynthetic genes by a $1 \mathrm{~kb}$ AT-rich $(\mathrm{G}+\mathrm{C} 45 \%)$ section that may represent a bidirectional promoter. The $t b l A, t a b A$, and $t a b B$ genes have been previously described [13 15]. Several genes in the block appear to be similar to genes in amino acid synthesis and modification pathways, reinforcing previous hypotheses that tabtoxin biosynthesis occurs via a shunt from endogenous amino acid synthesis in the cell [14 16].
The first gene product in the block as transcribed right to left, TabC (ORF 9, Fig. 2), contains motif homology to some zinc-binding proteins but is otherwise novel in the database; next (ORF 8, Fig. 2) is TblA, which has weak similarity to some methylases but is also novel; next (ORF 7, Fig. 2) is TabA, which has high similarity to diamopimelate decarboxylase (LysA); TabB (ORF 6, Fig. 2) is highly similar to succinyldiaminopimelate aminotransferase (DapD); TabD (ORF 5, Fig. 2) is an Aatlike aminotransferase; and finally TabP (ORF 4, Fig. 2), a putative zinc metallopeptidase of the M20 family of carboxypeptidases that seems likely to be responsible for the maturation event that converts tabtoxin to its toxic form of tabtoxinine- $\beta$-lactam.

\section{III) Right Biosynthetic Block}

The lightly shaded region of Fig. 2 represents the 'rightward' arm of the tabtoxin biosynthetic region. The first two genes in the region encode proteins similar to a pair known to be involved in the synthesis of clavulanic acid, a clavam antibiotic inhibitor of $\beta$-lactamases [17]. The first ORF (ORF 10, Fig. 2) in block III, TblS, has similarity to the $\beta$-lactam synthase, related to asparagine synthetases, that catalyzes the formation of the $\beta$-lactam ring in clavulanic acid [18]. The adjacent ORF, TblC, (ORF 11, 
Fig. 2) shows similarity to clavaminic acid synthase, related to taurine catabolism dioxygenases, which carries out three reactions in the clavulanic acid pathway [17]. The third gene in the block, TblD, (ORF 12, Fig. 2) appears to represent an interesting gene fusion whose product is a full-length GMC-oxidoreductase with a GNAT family acetyltransferase domain on the carboxyterminal end. The next ORF, TblE, (ORF 13, Fig. 2) in the block is a putative membrane protein of unknown function. It apparently forms a functional pair with its right-most neighbor, TblF, (ORF 14, Fig. 2), a presumptive enzyme with similarity to D-alanine-D-alanine ligase, in that the gene pair is also found together in at least Bradyrhizobium and Yersinia. The last gene (ORF 15, Fig. 2) in the block encodes a member of the major facilitator superfamily (MFS) of transporters and multidrug resistances [19], here designated TblR. Tabtoxin resistance could be conferred on mutants deleted for the toxin region by a $6.5 \mathrm{~kb} B a m \mathrm{HI}-B g l \mathrm{II}$ fragment containing the three rightmost ORFs in block III (Fig. 2). Resistance was also demonstrated with this fragment in JJR329, a nonpathogenic mutant of $P$. syringae pv. syringae, a pathovar that does not produce tabtoxin. This protein is most similar to the sugar transporter subgroup of the MFS, and is predicted to have 12 transmembrane helices by analysis with TMHMM (http://www.cbs.dtu.dk/ services/TMHMM-2.0/).

\section{IV) Gene Cluster Block}

After the stop signal for the multidrug resistance gene, there is a section of some $500 \mathrm{bp}$ in length with no identifiable ORFs, followed by a series of five genes (ORFs 16-20, Fig. 2) predicted to be transcribed with opposing polarity to the block III biosynthetic region. No firm functional assignment could be made for any of these genes, although two of them appear to be integral membrane proteins. Further investigation indicated that most or all of the genes in this block are usually found as a cluster with similar organization in a wide variety of bacteria, and are often located in pathogenicity or gene islands where such have been defined. The diverse list of species containing the cluster include Salmonella enterica (subspec. enterica serovar typhi), Rubrivivax gelatinosus, Azotobacter vinelandii, Xanthomonas campestris pv. campestris, and Ralstonia metallodurans. Interestingly, the entire cluster is also contained in the PAPI-1 pathogenicity island of $P$. aeruginosa PA14 (see above) as ORFs RL014018, where a mutation of the gene encoding RL016 caused a decrease in bacterial growth on Arabidopsis while not affecting virulence in mice [11]. The remaining $1 \mathrm{~kb}$ of the cosmid insert, while containing no identifiable ORFs, showed similarity at the nucleotide level to the integron-
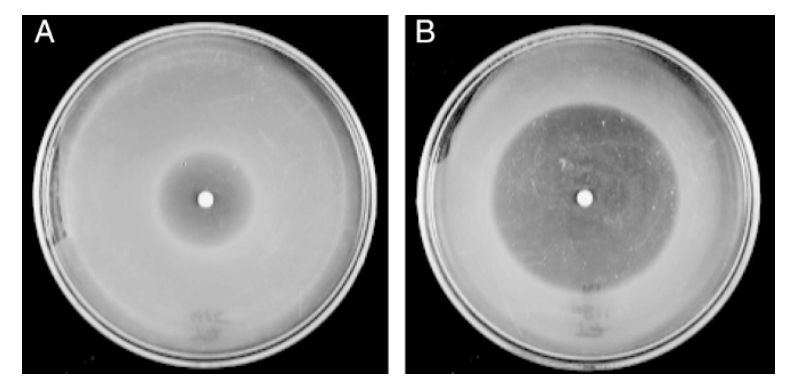

Fig. 3 The hypersensitivity phenotype associated with the $11 \mathrm{~kb}$ BamHI fragment is shown. One hundred microliters of a culture of tabtoxin deletion mutant Tox7 in King's B medium, containing either the plasmid vector pRK415 (Fig. 2A) or that vector with the BamHI fragment insert (Fig. 2B), were inoculated into an M9 agar overlay and then challenged with a paper disk containing semipurified tabtoxin [5].

rich plasmid pSCH884 of $P$. aeruginosa, as well as to a region of the $P$. syringae pv. tomato chromosome.

As shown in Fig. 2, both toxin production and toxin resistance were associated with the span of the region encompassing blocks II and III; this span also includes the prophage fragment that seems likely to be a vestigial remnant of a transfer event. Previous results had demonstrated that $\operatorname{Tn} 5$ insertions within the tabtoxin region resulted in the loss of toxin production without loss of host resistance to the antibiotic [5]. Sequence analysis confirms that the insertions in two of those mutants occurred in the $t a b C$ and $t b l S$ genes indicating that those two genes are required for antibiotic biosynthesis. Insertions in $t b l S$ could also be restored for toxin production by an EcoRI-HindIII fragment containing the $\beta$-lactam synthase gene by itself. This gene alone was not sufficient for toxin synthesis, as evidenced by the fact that its introduction did not confer toxin production on either deletion mutants or on JJR329. The ORF 15 tblR transporter gene would seem a strong candidate for being causal to resistance. However, some smaller clones with a complete MFS transporter showed no resistance to the toxin, possibly due to the lack of an independent promoter or to a requirement for assisting gene products. This might suggest that the other ORFs present on the restoring BamHI-BglII, ORF 13 and ORF 14, form an operon with ORF 15 and perhaps have a functional relationship as well. Attempts to express ORF 15 from a non-native lac promoter yielded only $E$. coli clones with the gene in opposing polarity to the promoter, suggesting that constitutive expression of the gene by itself was deleterious for the host cell.

An unexpected hypersensitivity phenotype was found associated with a Bam HI fragment containing the tabtoxin 
block II along with the two genes analogous to the clavulanic acid pathway (Fig. 3). This phenotype was not exhibited in strains with subclones containing the $t b l S$ gene by itself, suggesting that the phenotype might be resident in block II. This phenotype is reminiscent of a similar phenomenon observed previously with subclones containing only a portion of the mercury resistance operon. There, the expression of a high affinity sequestration protein MerT in the absence of the detoxifying mercuric reductase, encoded in the missing part of the operon, led to hypersensitivity to several mercury compounds $[20,21]$. The production of a high affinity cell component without the concomitant expression of the toxin resistance might explain this interesting phenotype.

\section{References}

1. Durbin RD, Uchytil TF, Steel JA, Rebeiro LD. Tabtoxininebeta-lactam from Pseudomonas tabaci. Phytochemistry 17: 147-148 (1978)

2. Levi C, Durbin RD. The isolation and properties of a tabtoxin-hydrolysing aminopeptidase from the periplasm of Pseudomonas syringae pv. syringae. Physiol Mol Plant Pathol 28: 345-352 (1986)

3. Sinden SL, Durbin RD. Glutamine synthetase inhibition: possible mode of action of wildfire toxin from Pseudomonas tabaci. Nature 229: 379-380 (1968)

4. Thomas MD, Durbin RD. Glutamine synthetase from Pseudomonas syringae pv. tabaci: properties and inhibition by tabtoxinine-beta-lactam. J Gen Microbiol 131: 1061-1067 (1985)

5. Kinscherf TG, Coleman RH, Barta TM, Willis DK. Cloning and expression of the tabtoxin biosynthetic region from Pseudomonas syringae. J Bacteriol 173: 4124-4132 (1991)

6. Ditta G, Stanfield S, Corbin D, Helinski DR. Broad host range DNA cloning system for gram-negative bacteria: construction of a gene bank of Rhizobium meliloti. Proc Natl Acad Sci USA 77: 7347-7351 (1980)

7. Arnold DL, Jackson RW, Fillingham AJ, Goss SC, Taylor JD, Mansfield JW, Vivian A. Highly conserved sequences flank avirulence genes: isolation of novel avirulence genes from Pseudomonas syringae pv. pisi. Microbiology 147: 1171-1182 (2001)

8. Jackson RW, Athanassopoulos E, Tsiamis G, Mansfield JW, Sesma A, Arnold DL, Gibbon MJ, Murillo J, Taylor JD, Vivian A. Identification of a pathogenicity island, which contains genes for virulence and avirulence, on a large native plasmid in the bean pathogen Pseudomonas syringae pathovar phaseolicola. Proc Natl Acad Sci USA 96: 10875-1080 (1999)

9. Kiewitz C, Larbig K, Klockgether J, Weinel C, Tummler B.
Monitoring genome evolution ex vivo: reversible chromosomal integration of a $106 \mathrm{~kb}$ plasmid at two tRNA(Lys) gene loci in sequential Pseudomonas aeruginosa airway isolates. Microbiology 146 ( Pt 10): 2365-2373 (2000)

10. Klockgether J, Reva O, Larbig K, Tummler B. Sequence analysis of the mobile genome island pKLC102 of Pseudomonas aeruginosa C. J Bacteriol 186: 518-534 (2004)

11. He J, Baldini RL, Deziel E, Saucier M, Zhang Q, Liberati NT, Lee D, Urbach J, Goodman HM, Rahme LG. The broad host range pathogen Pseudomonas aeruginosa strain PA14 carries two pathogenicity islands harboring plant and animal virulence genes. Proc Natl Acad Sci USA 101: 2530-2535 (2004)

12. Brussow H, Canchaya C, Hardt WD. Phages and the evolution of bacterial pathogens: from genomic rearrangements to lysogenic conversion. Microbiol Mol Biol Rev 68: 560-602, table of contents (2004)

13. Barta TM, Kinscherf TG, Uchytil TF, Willis DK. DNA sequence and transcriptional analysis of the tblA gene required for tabtoxin biosynthesis by Pseudomonas syringae. Appl Environ Microbiol 59: 458-466 (1993)

14. Liu L, Shaw PD. Characterization of dapB, a gene required by Pseudomonas syringae pv. tabaci BR2.024 for lysine and tabtoxinine-beta-lactam biosynthesis. J Bacteriol 179: 507-513 (1997)

15. Engst K, Shaw PD. Identification of a lysA-like gene required for tabtoxin biosynthesis and pathogenicity in Pseudomonas syringae pv. tabaci strain PTBR2.024. Mol Plant Microbe Interact 5: 322-329 (1992)

16. Unkefer CJ, London RE, Durbin RD, Uchytil TF, LangstonUnkefer PJ. The biosynthesis of tabtoxinine-beta-lactam. Use of specifically 13C-labeled glucose and 13C NMR spectroscopy to identify its biosynthetic precursors. J Biol Chem 262: 4994-4999 (1987)

17. McGowan SJ, Bycroft BW, Salmond GP. Bacterial production of carbapenems and clavams: evolution of betalactam antibiotic pathways. Trends Microbiol 6: 203-208 (1998)

18. Bachmann BO, Li R, Townsend CA. beta-Lactam synthetase: a new biosynthetic enzyme. Proc Natl Acad Sci USA 95: 9082-9086 (1998)

19. Saier MH, Jr., Beatty JT, Goffeau A, Harley KT, Heijne WH, Huang SC, Jack DL, Jahn PS, Lew K, Liu J, Pao SS, Paulsen IT, Tseng TT, Virk PS. The major facilitator superfamily. J Mol Microbiol Biotechnol 1: 257-279 (1999)

20. Brown NL, Shih YC, Leang C, Glendinning KJ, Hobman JL, Wilson JR. Mercury transport and resistance. Biochem Soc Trans 30: 715-718 (2002)

21. Foster TJ, Nakahara H, Weiss AA, Silver S. Transposon Agenerated mutations in the mercuric resistance genes of plasmid R100-1. J Bacteriol 140: 167-181 (1979) 